\title{
CYCLIC VECTORS AND IRREDUCIBILITY FOR PRINCIPAL SERIES REPRESENTATIONS. II $\left({ }^{1}\right)$
}

BY

\author{
NOLAN R. WALLACH
}

\begin{abstract}
This paper is a continuation of the author's paper Cyclic vectors and irreducibility for principal series representations. In this paper the nonunitary principal series is studied. Using a theorem of Kostant, a sufficient condition is found for irreducibility of nonunitary principal series representations.
\end{abstract}

1. Introduction. This paper is a continuation of the analysis of Wallach [6] of the principal series of representations using results of Kostant [4], [5]. In this paper we apply our techniques to get conditions leading to irreducibility for nonunitary principal series representations.

Our main new tool is Theorem 2.1 of this paper (which we think is new) which says (in essence) that if a representation $(\pi, H)$ of a semisimple group $G$ (not necessarily unitary) has the same character as an infinitesimally irreducible representation of $G,\left(\pi_{1}, H^{\prime}\right)$ then $(\pi, H)$ and $\left(\pi_{1}, H^{\prime}\right)$ are infinitesimally equivalent. We apply this result to the nonunitary principal series by using Harish-Chandra's computation of the characters of the nonunitary principal series (see [2], and Theorem 3.1 in this paper).

We are indebted to Professor B. Kostant for giving us access to the manuscript of [5].

2. A result on characters. Let $G$ be a connected semisimple Lie group with finite center. Let $K$ be a maximal compact subgroup of $G$. Let $\hat{K}$ be the set of all equivalence classes of irreducible finite-dimensional representations of $K$. A representation $(\pi, H)$ of $G$ on a separable Hilbert space (a representation will mean a continuous representation, that is, the map $g, f \mapsto \pi(g) f$ of $G \times H$ to $H$ is continuous) is said to be admissible if

(1) as a $K$-representation, $(\pi, H)$ is unitary,

(2) as a $K$-representation, $H$ splits into a direct sum $H=\sum_{\gamma \in \hat{K}} H_{y}$ where $H_{\gamma}$ is a direct sum of $m_{\gamma}$ copies of an element of $\gamma$ in $\hat{K}$ and $m_{\gamma} \leqq C d_{\gamma}$ where $d_{\gamma}$ is the dimension of any element of $\gamma$.

Received by the editors March 12, 1971.

AMS 1969 subject classifications. Primary 2260, 2257.

Key words and phrases. Representation, Hilbert space, character, universal enveloping algebra, irreducible, admissible, Haar measure, trace class, module, root, Weyl group, PeterWeyl Theorem.

(1) Supported by NSF Grant GP-27959. 
If $(\pi, H)$ is admissible and if $C_{0}^{\infty}(G)$ denotes the space of all complex valued $C^{\infty}$ functions on $G$ with compact support then if $f \in C_{0}^{\infty}(G)$ and if $d x$ is Haar measure on $G$ define

$$
\pi(f)=\int_{G} f(x) \pi(x) d x .
$$

Then $\pi(f)$ as an operator on $H$ is of trace class (see Harish-Chandra [1]). That is, if $\left\{\psi_{n}\right\}$ is an orthonormal basis of $H$ then

$$
\Theta_{\pi}(f)=\sum_{n=1}^{\infty}\left\langle\pi(f) \psi_{n}, \psi_{n}\right\rangle
$$

is finite and independent of the basis $\left\{\psi_{n}\right\}(<$,$\rangle is the Hilbert space inner product$ on $H$ ).

$\Theta_{\pi}$ is called the character of $(\pi, H)$.

Following Harish-Chandra we define, for $\gamma \in R$, the function

$$
\phi_{\gamma}^{\pi}(g)=\operatorname{tr} E_{\gamma}^{\pi} \pi(g) E_{\gamma}^{\pi}
$$

where $E_{\gamma}^{\pi}: H \rightarrow H_{\gamma}$ is the orthogonal projection. We note that if $\chi_{\gamma}$ is the character of $\gamma \in \hat{K}$ then

$$
E_{\gamma}^{\pi} v=d(\gamma) \int_{K} \chi_{\gamma}(k) \pi(k) v d k,
$$

where $d k$ is normalized Haar measure on $K$.

LEMMA 2.1. If $\left(\pi_{1}, H^{1}\right)$ and $\left(\pi_{2}, H^{2}\right)$ are admissible representations of $G$ so that $\Theta_{\pi_{1}}=\Theta_{\pi_{2}}$ then, for each $\gamma \in \hat{R}, \phi_{\gamma}^{\pi_{1}}=\phi_{\gamma}^{\pi_{2}}$.

Proof. Suppose for some $\gamma \in \hat{K}, \phi_{\gamma}^{\pi_{1}}-\phi_{\gamma}^{\pi_{2}}=\phi \neq 0$. Then there is $f \in C_{0}^{\infty}(G)$ so that $\int_{G} \phi(g) f(g) d g \neq 0$. Setting $f^{\prime}(x)=d_{\gamma} \int_{K} \chi_{\gamma}(k) f\left(k^{-1} x\right) d k$ a computation shows that $\Theta_{\pi_{1}}\left(f^{\prime}\right)-\Theta_{\pi_{2}}\left(f^{\prime}\right)=\int \phi(g) f(g) d g$. This contradiction yields the result.

If $(\pi, H)$ is admissible let $H_{F}$ be the algebraic direct sum of the $H_{\gamma}$. Then $H_{F}$ is the space of all $f \in H$ so that $\pi(K) f$ is contained in a finite-dimensional subspace of $H$.

Let $\mathrm{g}$ be the lie algebra of $G$ and let $U(\mathfrak{g})$ be the complexified universal enveloping algebra of $\mathrm{g}$. If $X \in \mathfrak{g}, f \in H_{F}$, define $\pi(X) f=\left.(d / d t) \pi(\exp (t X)) f\right|_{t=0}$. Then $\pi(X) H_{F} \subset H_{F}$ and defines a representation of $U(\mathrm{~g})$ on $H_{F}$. We say that $(\pi, H)$ is infinitesimally irreducible if the representation $\left(\pi, H_{F}\right)$ is an irreducible representation of $U(\mathrm{~g})$. If $\left(\pi_{1}, H^{1}\right)$ and $\left(\pi_{2}, H^{2}\right)$ are admissible representations of $G$ then $\pi_{1}$ is said to be infinitesimally equivalent with $\pi_{2}$ if the representations $\left(\pi_{1}, H_{F}^{1}\right)$ and $\left(\pi_{2}, H_{F}^{2}\right)$ are equivalent representations of $U(\mathfrak{g})$.

THEOREM 2.1. Let $\left(\pi_{1}, H^{1}\right)$ and $\left(\pi_{2}, H^{2}\right)$ be admissible representations of $G$. Suppose that $\left(\pi_{1}, H^{1}\right)$ is infinitesimally irreducible and that $\Theta_{\pi_{1}}=\Theta_{\pi_{2}}$. Then $\left(\pi_{1}, H^{1}\right)$ and $\left(\pi_{2}, H^{2}\right)$ are infinitesimally equivalent. 
Proof. For each $v \in H_{F}^{i}$ define $A_{v}^{i}: H_{F}^{i} \rightarrow C^{\infty}(G)$ by

$$
A_{v}^{i}(w)(g)=\left\langle\pi_{i}(g)^{-1} w, v\right\rangle .
$$

Then $A_{v}^{i}\left(\pi_{i}(X) w\right)(g)=\left.(d / d t) A_{v}^{i}(w)(\exp (-t X) g)\right|_{t=0}$. Thus if we let $U(g)$ act on $C^{\infty}(G)$ by $X \cdot f(g)=\left.(d / d t) f(\exp (-t X) g)\right|_{t=0}$ for $X \in g, A_{v}^{i}$ is a homomorphism of $U(\mathrm{~g})$-representations.

Let $\gamma \in \hat{K}$ be so that $\phi_{\gamma}^{\pi} \neq 0$. Let $C_{\gamma}^{i}=\operatorname{dim} H_{\gamma}^{i}$. Then Lemma 2.1 implies that $C_{\gamma}^{1}=C_{\gamma}^{2}$ (indeed $C_{\gamma}^{i}=\phi_{\gamma}^{\pi_{i}}(e)$ ). Let $C_{\gamma}=C_{\gamma}^{1}$.

Let $v_{1}^{i}, \ldots, v_{C_{\gamma}}^{i}$ be an orthonormal basis of $H_{\gamma}^{i}, i=1,2$. Let $\eta: G \rightarrow G$ be defined by $\eta(g)=g^{-1}$. Then

$$
\phi_{\gamma}^{\pi_{i} \circ \eta}=\sum_{j=1}^{C_{y}} A_{v_{j}^{i}}^{i}\left(v_{j}^{i}\right) .
$$

Now $A_{v_{j}^{1}}^{1}\left(H_{F}^{1}\right)$ is equivalent with $H_{F}^{1}$ as a $U(\mathrm{~g})$-module for $j=1, \ldots, C_{\gamma}$, and

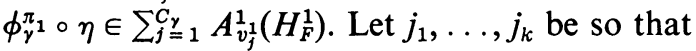

(a) $\phi_{\gamma}^{\pi} \circ \eta \in \sum_{i=1}^{k} A_{v_{j i}^{1}}^{1}\left(H_{F}^{1}\right)$,

(b) no subset of $\left\{j_{1}, \ldots, j_{k}\right\}$ satisfies (a).

It is then clear that if $V_{i}=A_{v_{1}^{1}}^{1}\left(H_{F}^{1}\right)$ then the sum $V_{1}+\cdots+V_{k}$ is direct (indeed $V_{j}$ is an irreducible $U(\mathrm{~g})$-module). Set $V=V_{1} \oplus \cdots \oplus V_{k}$. Let $P_{j}: V \rightarrow V_{j}$ be the corresponding projection.

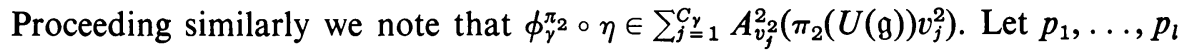
be so that

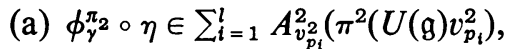

(b) no subset of $\left\{p_{1}, \ldots, p_{l}\right\}$ satisfies (a).

Let

$$
W_{i}=A_{v_{p_{i}}}^{2}\left(\pi_{2}(U(\mathfrak{g})) v_{p_{i}}^{2}\right), \quad i=1, \ldots, l .
$$

Then $W_{i}$ is a $U(\mathrm{~g})$-submodule of $C^{\infty}(G)$ and is equivalent to a subquotient of $H_{F}^{2}$. Let $W=W_{1}+\cdots+W_{l}$. Let $W^{j}=\sum_{k=j}^{l} W_{k}$. Let $Q_{j}: W^{j} \rightarrow W^{j} / W^{j+1}$ be the natural map.

By hypothesis $W \cap V \neq(0)$. Suppose that $\left.\operatorname{Ker} Q_{1}\right|_{W \cap V}=(0)$. Then $W \cap V$ as a $U(\mathrm{~g})$-module is equivalent to a subquotient of $W_{1}$, hence $W \cap V$ is equivalent to a subquotient of a subquotient of $H_{F}^{2}$. Now for some $j, P_{j}(W \cap V) \neq(0)$. Thus $P_{j}(W \cap V)=V_{j}$ since $V_{j}$ is irreducible. But $V_{j}$ is equivalent to $H_{F}^{1}$. Thus $H_{F}^{1}$ is equivalent to a quotient of a subquotient of a subquotient of $H_{F}^{2}$. But as $K$-representations $H_{F}^{1}$ and $H_{F}^{2}$ are equivalent. Hence $H_{F}^{1}$ is equivalent to $H_{F}^{2}$ as a $U(\mathfrak{g})$ representation. Thus if $\left.\operatorname{Ker} Q_{1}\right|_{W \cap V}=(0)$ the theorem is proved.

Otherwise $Z_{1}=\left.\operatorname{Ker} Q_{1}\right|_{W \cap V} \neq(0) . Z_{1} \subset W^{2} \cap V$. If $\left.\operatorname{Ker} Q_{2}\right|_{z_{1}}=0$ then applying to the argument above we see that $H_{F}^{1}$ and $H_{F}^{2}$ are equivalent as $U(\mathrm{~g})$-representations. Otherwise $Z_{2}=\operatorname{Ker}\left(\left.Q_{2}\right|_{Z_{1}}\right) \neq 0, Z_{2} \subset W^{3} \cap V$. Continuing this process we either prove the theorem or after $l-1$ stages we find $W_{l} \cap V \neq(0)$. But then for some $j, P_{j}\left(W_{l} \cap V\right) \neq(0)$. Thus a quotient of $W_{l} \cap V$ is equivalent to $H_{F}^{2}$. But 
$W_{l} \cap V$ is equivalent to a subquotient of $H_{F}^{1}$. Thus noting that as $K$-representations $H_{F}^{1}$ and $H_{F}^{2}$ are equivalent we have completely proved the theorem.

Note. The condition (1) of admissibility can be removed and the formula (*) can be used to define $E_{\gamma}^{\pi}$ for a representation satisying (2).

Corollary 2.1 (to the proof of Theorem 2.1). Let $\left(\pi_{1}, H^{1}\right)$ and $\left(\pi_{2}, H^{2}\right)$ be admissible representations of $G$. Suppose that

(1) $\left(\pi_{1}, H^{1}\right)$ is infinitesimally irreducible,

(2) as $K$-representations $\left(\pi_{1}, H^{1}\right)$ and $\left(\pi_{2}, H^{2}\right)$ are equivalent,

(3) there is $\gamma \in \hat{K}$ so that $\phi_{\gamma}^{\pi_{1}} \neq 0$ and $\phi_{\gamma}^{\pi_{1}}=\phi_{\gamma}^{\pi_{2}}$.

Then $\left(\pi_{1}, H^{1}\right)$ and $\left(\pi_{2}, H^{2}\right)$ are infinitesimally equivalent.

3. The principal series. Let $G, K$ be as in $\S 2$. Let $G=K A N$ be an Iwasawa decomposition of $G$. Let $M$ be the centralizer of $A$ in $K$. Let $M^{*}$ be the normalizer of $A$ in $K$. Then $M^{*} / M$ defines a finite group of automorphisms of $A$, the Weyl group, $W$, of $A$. Let $\hat{M}$ be the set of all equivalence classes of irreducible finite-dimensional representations of $M$. If $\xi \in \hat{M}$ we fix an element $\left(\xi, H_{\xi}\right)$ of $\xi$. Let $a$ be the Lie algebra of $A$, and $\mathfrak{a}^{*}, \mathfrak{a}_{C}^{*}$ be the spaces of linear real-valued and complex-valued forms on $a$. If $s \in W$, and $m^{*} \in s, \xi \in \hat{M}, m \in M$ we define $\xi^{s}(m)=\xi\left(m^{*-1} m m^{*}\right)$. If $\nu \in \mathfrak{a}_{C}^{*}$ we define $s v(H)=\nu\left(\operatorname{Ad}\left(m^{*}\right)^{-1} H\right)$.

If $\xi \in \hat{M}, \nu \in \mathfrak{a}_{C}^{*}$ we define an admissible representation of $G,\left(\pi_{\xi, v}, H^{\xi, v}\right)$ as follows:

(1) $H^{\xi, v}$ is the space of all measurable functions, $f$, from $G$ to $H_{\xi}$ so that

(i) $f($ gman $)=\xi(m)^{-1} e^{-v(\log a)} f(g)(\log : A \rightarrow \mathfrak{a}$ is the universe to exp: $\mathfrak{a} \rightarrow A)$.

(ii) $\int_{K}\|f(k)\|^{2} d k<\infty$,

(2) $\left(\pi_{\xi, v}\left(g_{0}\right) f\right)(g)=f\left(g_{0}^{-1} g\right)$.

It is easy to see that $\left(\pi_{\xi, v}, H^{\xi, v}\right)$ is an admissible representation of $G$ (indeed Frobenius reciprocity implies condition (2) of admissibility).

Let $\Theta_{\xi, v}$ be the character of $\left(\pi_{\xi, v}, H^{\xi, v}\right)$.

TheOREM 3.1 (HARISH-ChANDRA [2]). Let $\rho \in \mathfrak{a}^{*}$ be defined by

$$
\rho(H)=\frac{1}{2} \operatorname{tr}\left(\left.\operatorname{ad} H\right|_{\mathfrak{n}}\right)
$$

(n the Lie algebra of $N$ ). If $\xi, \xi^{\prime} \in \hat{M}, \nu, \nu^{\prime} \in \mathfrak{a}_{C}^{*}$ then $\Theta_{\xi, v}=\Theta_{\xi^{\prime}, v^{\prime}}$ if and only if there is $s \in W$ so that $\xi^{s}=\xi^{\prime}, s(\nu-\rho)+\rho=\nu^{\prime}$.

Actually in [2], Harish-Chandra has a different definition of the principal series. The proof he gives, however, goes through without change to our definition of the principal series.

We note that with our definition of the principal series the unitary principal series is the set of all $\pi_{\xi, \rho+\sqrt{ }(-1) v}$, where $\xi \in \hat{M}, v \in \mathfrak{a}^{*}$, indeed,

LEMMA 3.1. There is a nondegenerate, sesquilinear pairing, ( , ), between $H^{\xi, v}$ and $H^{\xi, 2 \rho-\bar{v}}$ for each $\xi \in \hat{M}, \nu \in \mathfrak{a}_{C}^{*}$ so that if $f_{1} \in H^{\xi, \nu}, f_{2} \in H^{\xi, 2 \rho-\bar{v}}, g \in G$, then

$$
\left(\pi_{\xi, v}(g) f_{1}, \pi_{\xi, 2 \rho-\bar{v}}(g) f_{2}\right)=\left(f_{1}, f_{2}\right)
$$


Proof. We use the integral formula that if $g \in G, g=k a n$ and $H(g)=\log a$ and if $f$ is a continuous function on $K$ then

$$
\int_{K} f(k(x k)) \exp [2 \rho(H(x k))] d k=\int_{K} f(k) d k
$$

(cf. Helgason [3]). The result is an immediate consequence of (1).

4. Extendible representations of $M$. We retain the notation of $\S 3$. We assume that $G$ is a linear Lie group. Let $\theta$ be the Cartan involution of $G$ corresponding to $K$. Let $\bar{N}=\theta(N)$. If $V$ is a finite-dimensional $G$-module and $H$ is a subgroup of $G$ define $V^{H}=\{v \in V \mid h \cdot v=v$ for all $h \in H\}$.

We say that $\xi \in \hat{M}$ is extendible if there is a finite-dimensional irreducible $G$ module, $V$, so that as an $M$-representation $V^{N}$ is in $\xi . V$ is called an extension of $\xi$. In Wallach [6] we proved

THEOREM 3.1. If $\xi \in \hat{M}$ there is $s \in W(A)$ so that $\xi^{s}$ is extendible.

Theorem 4.2. Let $\xi \in \hat{M}, \nu \in \mathfrak{a}_{C}^{*}$. There is at most one nonzero finite-dimensional $G$-invariant subspace of $H^{\xi, \nu}$. Denote this subspace by $V_{\xi, v}$ if it exists. Let $V_{\xi, \nu}=(0)$ if $H^{\xi, v}$ contains no nonzero finite-dimensional $G$-invariant subspace. If $V_{\xi, \nu} \neq(0)$ then $V_{\xi, v}$ is an extension of $\xi$. If $\xi \in \hat{M}$ there is $s \in W(A), \lambda \in \mathfrak{a}^{*}$ so that $V_{\xi^{s}, \lambda} \neq(0)$.

Proof. Suppose $V$ is a nonzero finite-dimensional subspace of $H^{\xi, v}$. Then applying Lie and Engel's theorem, $V^{\bar{N}} \neq(0)$. If $f \in V^{\bar{N}}$ then

$$
f(\bar{n} m a n)=\xi(m)^{-1} e^{-v(\log a)} f(e) .
$$

Thus since $\bar{N} M A N$ is open and dense in $G$ (cf. Helgason [3]) the map $V^{\bar{N}} \rightarrow H_{\xi}$ given by $f \mapsto f(e)$ is injective. Furthermore, if $m \in M$ then $\left(\pi_{\xi, v}(m) \cdot f\right)(e)=\xi(m) \cdot f(e)$. Thus the map $V^{N} \rightarrow H_{\xi}$ is surjective, hence it is bijective. This proves the uniqueness and that if $V_{\xi, v} \neq(0)$ then $V_{\xi, v}$ is an extension of $\xi$.

Let $\xi \in \hat{M}$; then by Theorem 4.1 there is $s \in W(A)$ so that $\xi^{s}$ is extendible. Let $V$ be an extension of $\xi^{s}$. In Wallach [6] it was shown that if $V^{*}$ is the contragradient representation to $V$, then $V^{N}$ is equivalent to $\left(V^{* N}\right)^{*}$ as an $M A$ module. If $v \in V$ define $\alpha(v)(g) \in\left(V^{* N}\right)^{*}=H_{\xi^{s}}$ by $\alpha(v)(g)(\mu)=\mu\left(g^{-1} v\right)$. Let $a \in A, v \in V^{N}$, $\lambda \in \mathfrak{a}^{*}$ be defined by $a \cdot v=e^{\lambda(\log a)} v$. Then it is easy to see that $\alpha(v)(g m a n)$ $=\xi(m)^{-1} e^{-\lambda(\log a)} \alpha(v)(g)$ (see Wallach [6]). This proves the result.

If $\nu \in \mathfrak{a}_{C}^{*}$ define $1_{\nu}(g)=\exp [-\nu(H(g))]$ for $g \in G$. Let $1_{\nu} \in H^{1, \nu}$. If $\xi \in \hat{M}$ let $\Omega_{\xi}=\left\{\lambda \in \mathfrak{a}^{*} \mid V_{\xi, \lambda} \neq(0)\right\}$.

Proposition 4.1. Let $\xi \in \hat{M}, \lambda \in \Omega_{\xi}$. If $\nu \in \mathfrak{a}_{C}^{*}$ and if $1_{v-\lambda}$ is a cyclic vector for $H^{1, v-\lambda}$ and if $f \in V_{\xi, \lambda}^{\bar{N}}, f \neq 0$ then $1_{\nu-\lambda} \cdot f$ is a cyclic vector for $H^{\xi, \nu}$.

For a proof of this result see Wallach [6].

The following result is a slight refinement of Theorem 3.3 of Wallach [6]. 
ThFOREM 4.3. Let $\xi \in \hat{M}, \lambda \in \Omega_{\xi}$. Suppose that as an $M$-representation the multiplicity of $\left(\xi, H_{\xi}\right)$ in $V_{\xi, \lambda}$ is one. If $\nu \in \mathfrak{a}_{C}^{*}$ and if $1_{\nu-\lambda}$ and $1_{2 \rho-\bar{\nu}-\lambda}$ are cyclic vectors for $H^{1, v-\lambda}$ and $H^{1,2 \rho-\bar{v}-\lambda}$ then $H^{\xi, v}$ is irreducible.

Proof. We observe that by Frobenius reciprocity the multiplicity of the $K$ representation $V_{\xi, \lambda}$ in $H^{\xi, v}$ as a $K$-representation is one. Suppose now that $U \subset H^{\xi, v}$ is a closed invariant subspace of $H^{\xi, \nu}$. Then either $1_{\nu, \lambda} \cdot V_{\xi, \lambda} \subset U$ or $1_{2 \rho-\bar{v}-\lambda} \cdot V_{\xi, \lambda}$ $\subset U^{\perp} \subset H^{\xi, 2 \rho-\bar{v}}$ (here " $\perp$ " is relative to the pairing of Lemma 3.1). Now Proposition 4.1 implies $U=H^{\xi, \nu}$ or $U^{\perp}=H^{\xi, 2 \rho-\bar{\nu}}$. Hence $U=H^{\xi, \nu}$ or $U=(0)$. Q.E.D.

5. A theorem of Kostant. Let us retain the notation of the previous section. Let for $\phi \in \mathfrak{a}^{*}, \mathfrak{n}_{\phi}=\{x \in \mathfrak{n} \mid[h, x]=\phi(h) x$ for all $h \in \mathfrak{a}\}$. Let $\Lambda^{+}=\left\{\phi \in \mathfrak{a}^{*} \mid \mathfrak{a}_{\phi} \neq(0)\right\}$. Let $h_{\phi} \in \mathfrak{a}$ be defined by $\left\langle h_{\phi}, h\right\rangle=\phi(h)$ for $h \in \mathfrak{a},\langle$,$\rangle is the Killing form of \mathrm{g}$. Let $\Lambda_{1}^{+}=\left\{\phi \in \Lambda^{+} \mid \phi / 2 \notin \Lambda^{+}\right\}$. If $\phi \in \Lambda_{1}^{+}$, set $n_{\phi}=1$ if $2 \phi \notin \Lambda^{+}, n_{\phi}=2$ if $2 \phi \in \Lambda^{+}$. Let $m_{\phi}=(\operatorname{dim} \phi) / 2$ if $2 \phi \notin \Lambda^{+}$and $m_{\phi}=(\operatorname{dim} \phi) / 2+1$ if $2 \phi \in \Lambda^{+}$.

TheOREM 5.1 (Kostant [5]). Let $\lambda \in \mathfrak{a}_{C}^{*}$ then $1_{\lambda}$ is not a cyclic vector for $H^{1, \lambda}$ if and only if

$$
(\lambda-\rho)\left(h_{\phi}\right) / \phi\left(h_{\phi}\right)+m_{\phi}=n_{\phi} k
$$

where $k$ is a nonpositive integer.

We apply the above theorem of Kostant combined with our previous results to the case $G$ is a complex semisimple group. In this case $K$ is a compact form of $G$. $M$ is a maximal torus of $K$ and $M=\exp (\sqrt{ }(-1) \mathfrak{a})$. Thus if $\xi \in \hat{M}$ and if $\dot{\xi}$ is the differential of $\xi$ then $\dot{\xi}$ induces a linear form $\dot{\xi} \in \mathfrak{a}^{*}$. Furthermore if $\dot{\xi}$ is negative integral $\left(2 \dot{\xi}\left(h_{\phi}\right) / \phi\left(h_{\phi}\right)\right.$ a nonpositive integer for each $\left.\phi \in \Lambda^{+}\left(=\Lambda_{1}^{+}\right)\right)$then $V_{\xi, \xi}$ is the holomorphic irreducible finite-dimensional representation of $G$ with lowest weight $\xi$.

THEOREM 5.2. (Compare with Zelobenko [7].) Let $G$ be a complex semisimple Lie group. Let $\xi \in \hat{M}, v \in \mathfrak{a}_{C}^{*}$. $\left(\pi_{\xi, v}, H^{\xi, v}\right)$ is irreducible if there is $s \in W(A)$ so that $s \cdot \dot{\xi}$ is negative integral and if for each $\phi \in \Lambda^{+}$

$$
s(\nu-\dot{\xi}-\rho)\left(h_{\phi}\right) / \phi\left(h_{\phi}\right)+1 \text { and } s(\rho-\bar{\nu}-\dot{\xi})\left(h_{\phi}\right) / \phi\left(h_{\phi}\right)+1
$$

are not nonpositive integers.

Proof. Suppose $s$ is so that the conditions of the theorem are satisfied. Then noting that $m_{\phi}=n_{\phi}=1$ for $\phi \in \Lambda^{+}$and that the multiplicity of the character $\xi^{s}$ in $V_{\xi^{s}, s \cdot \xi^{*}}$ is one, the conditions of the theorem and Theorems 5.1, 4.3 imply that $H^{\xi^{s}, s(v-\rho)+\rho}$ is irreducible. But $\Theta_{\xi, v}=\Theta_{\xi^{s}, s(v-\rho)+\rho}$, by Theorem 3.1. Hence the result follows from Theorem 2.1 .

We now apply the results to $G=\mathrm{SL}(n, R)$. In this case $K=\mathrm{SO}(n), A$ is the group of all diagonal matrices with positive entries and determinant 1. $M$ is the group of 
all diagonal matrices with entries \pm 1 and determinant one. If $m \in M$ then

$$
m=\left[\begin{array}{ccc}
m_{1} & & 0 \\
0 & \ddots & \\
& & m_{n}
\end{array}\right]
$$

set $\varepsilon_{i}(m)=m_{i}$. Let $\varepsilon_{0}(m)=1$ for all $m \in M$. Then every element of $\hat{M}$ is of the form $\varepsilon_{0} \varepsilon_{i_{1}} \cdots \varepsilon_{i_{r}}$ with $0<i_{1}<\cdots<i_{r} \leqq n-1$. Let $\xi_{r}=\varepsilon_{0} \varepsilon_{1} \cdots \varepsilon_{r}, r=0, \ldots, n-1$. Let $V^{0}=C$ with $G$ acting trivially. Let $V^{1}=C^{n}$ with $G$ acting in the usual (matrix) fashion on $C^{n}$. Let $V^{r}=\Lambda^{r} V^{1}, r=1, \ldots, n-1$. Then $V^{n-r}$ is an extension of $\xi_{r}$. Let $\lambda_{r}$ be the action of $A$ on $\left(V^{n-r}\right)^{N}(\bar{N}$ is the group of all lower triangular matrices with ones on the diagonal). Then

$$
\lambda_{r}\left[\begin{array}{ccc}
a_{1} & & 0 \\
0 & \ddots & \\
& & a_{n}
\end{array}\right]=a_{r+1} \cdots a_{n} .
$$

$\mathfrak{a}$ is the set of all diagonal $n \times n$ real matrices with trace 0 . Let for $h$ in $\mathfrak{a}$

$$
h=\left[\begin{array}{ccc}
h_{1} & & 0 \\
0 & \ddots & \\
& & h_{n}
\end{array}\right],
$$

$\phi_{i}(h)=h_{i}$. Then $a_{C}^{*}=\left\{\sum c_{i} \phi_{i} \mid \sum c_{i}=0\right\} . \lambda_{r}(a)=\exp \left[\lambda_{r}(\log a)\right]$ where $\lambda_{r}=\sum_{j=1}^{n-r} \phi_{r+j} \mid \mathfrak{a}$. $\rho=-\left.\sum_{r=1}^{n-1} \lambda_{r}\right|_{\mathfrak{a}}=\left.\sum_{j=1}^{n-1}(n-j) \phi_{j}\right|_{\mathfrak{a}}$.

THEOREM 5.3. Let $G=\operatorname{SL}(n, R)$.

(1) Suppose $n=2 p+1, p$ a positive integer. Let $\xi \in \hat{M}, \nu \in \mathfrak{a}_{C}^{*}$. If there is an $s \in W(A)(W(A)$ acts by permuting entries in $M$ and in $A)$ so that $s \cdot \xi=\xi_{r}$ and if

$$
\left(s(\nu-\rho)+\lambda_{n-r}\right)\left(h_{\phi}\right) / \phi\left(h_{\phi}\right)+\frac{1}{2}
$$

and

$$
\left(s(\rho-\bar{\nu})+\lambda_{n-r}\right)\left(h_{\phi}\right) / \phi\left(h_{\phi}\right)+\frac{1}{2}
$$

are not nonpositive integers then $\left(\pi_{\xi, v}, H^{\xi, v}\right)$ is irreducible.

(2) Suppose that $n=2 p, p$ a positive integer. If there is $s \in W(A)$ so that $s \cdot \xi=\xi_{r}$ and $r \neq p$, and if

$$
\left(s(\nu-\rho)+\lambda_{n-r}\right)\left(h_{\phi}\right) / \phi\left(h_{\phi}\right)+\frac{1}{2}
$$

and

$$
\left(s(\rho-\bar{\nu})+\lambda_{n-r}\right)\left(h_{\phi}\right) / \phi\left(h_{\phi}\right)+\frac{1}{2}
$$

are not nonpositive integers then $\left(\pi_{\xi, v}, H^{\xi, v}\right)$ is irreducible.

Proof. The multiplicity if $\xi_{r}$ in $V^{n-r}$ is 1 in each of the above cases (1) or (2). $n_{\phi}=1$ and $m_{\phi}=\frac{1}{2}$ for all $\phi \in \Lambda^{+}\left(=\Lambda_{1}^{+}\right)$. Thus Theorem 5.1 combined with Theorem 4.3 implies that $H^{\xi s, s(v-\rho)+\rho}$ is irreducible. Since $\Theta_{\xi, \nu}=\Theta_{\xi^{8}, s(v-\rho)+\rho}$, Theorem 2.1 implies the result. 


\section{REFERENCES}

1. Harish-Chandra, Representations of semisimple Lie groups. III, Trans. Amer. Math. Soc. 76 (1954), 234-253. MR 16, 11.

2. - The Plancherel formula for complex semisimple Lie groups, Trans. Amer. Math. Soc. 76 (1954), 485-528. MR 16, 111.

3. S. Helgason, Lie groups and symmetric spaces, Battelle Rencontres. 1967 Lectures in Math. and Phys., Benjamin, New York, 1968, pp. 1-71. MR 38 \#4622.

4. B. Kostant, On the existence and irreducibility of certain series of representations, Bull. Amer. Math. Soc. 75 (1969), 627-642. MR 39 \#7031.

5. ——, (to appear).

6. N. Wallach, Cyclic vectors and irreducibility for principal series representations, Trans. Amer. Math. Soc. 158 (1971), 107-113.

7. D. Želobenko, The analysis of irreducibility in the class of elementary representations of $a$ semisimple complex Lie group, Izv. Akad. Nauk SSSR Ser. Mat. 32 (1968), 108-133= Math. USSR Izv. 2 (1968), 105-128. MR 37 \#2906.

Department of Mathematics, Rutgers University, New Brunswick, New Jersey 08903 